\title{
A study of changes in cognitive evoked potentials in persons with visual impairment
}

\author{
Anton Selskii ${ }^{1}$, Maksim Zhuravlev $^{1,2}$, Anastasiia Runnova ${ }^{1,2}$, Elena Grinina ${ }^{1}$, Marina \\ Konovalova $^{1}$, and Rail Shamionov ${ }^{1, *}$ \\ ${ }^{1}$ National Research Saratov State University named after N.G. Chernyshevsky, 83 Astrakhanskaya \\ str., 410012, Saratov, Russia \\ ${ }^{2}$ Saratov State Medical University named after V.I. Razumovsky, 112, Bolshaia Kazahia str. 410012, \\ Saratov, Russia
}

\begin{abstract}
In this work we have used psychophysiological assessments of the human brain electrical activity according to the classical neurological method for calculating the evoked potential. The experiment was designed to extraction cognitive evoked potentials. Taking into account the characteristic components, the temporal dynamics of the EEG data channels was investigated. This approach allows one to consistently assess the distribution of all components of the evoked potential on the subject's head map. Based on the results of evoked potentials processing, a statistical comparison of the components of evoked potentials in subjects of different groups by channels was carried out in accordance with the Wilcoxon test. Demonstrated for which channels the results significantly differ between the two groups of subjects. The sequence of evoked potential analysis demonstrated in the article suits for adjusting the settings of the "braincomputer" systems for a particular subject and allows to select channels used in further BCI training efficiently.
\end{abstract}

\section{Introduction}

Study of the human brain is one of the most important tasks of modern science. However, in order to study brain activity productively, scientists need an effective approach to measure activity, as well as a numerical method, which corresponds to any individual task. At present, there is a large number of methods, which allow to assess and measure activity of brain sections, e.g. encephalograms (EEG, MEG), functional MRI and computed tomography [13]. Each method is suitable for a certain range of tasks. In the study we used electroencephalogram, since this method is easy to use, non-invasive and inexpensive compared to others. At the same time, spatial and temporal EEG resolution is sufficient for the task of studying the evoked potentials (EP) and singling out individual psychophysiological characteristics from them [4]. After EEG data is obtained during the experiment, it is processed using one of the mathematical methods, the number of which is currently very large [5 - 9]. In this article the EEG data analysis was carried out through building cognitive evoked potentials [4, $10-14]$. This method is frequently used for analysis

\footnotetext{
* Corresponding author: selskiiao@gmail.com
} 
of EEG data experiments, where the subject is performing repetitive tasks, which require attention or cognitive activity. Apart from fundamental studies of the cognitive mechanisms of the human brain, evoked potentials are advantageous for the development of the "braincomputer interface" (BCI) systems, which are used for teaching or rehabilitating patients with various illnesses. In case of this kind of practical application, it is extremely important to take into consideration individual traits of the subject. Cognitive evoked potentials are typically characterized by the presence of the well-marked component $\mathrm{P}(300)[15,16]$. This component is present in evoked potentials when cognitive tasks are solved, and can be stably observed in a rather narrow time interval after stimulus presentation. However, despite the stability, individual characteristics (especially in case of strong suppression of cognitive abilities) can lead to a significant change in the observed evoked potentials, which must be taken into account when setting up the "brain-computer" system.

The purpose of this work is to study the diagnostic possibility of individual peculiarities in primary school children with impaired vision, based on the psychophysiological assessments. Experimental work and statistical analysis that were carried out allow to evaluate differences in spatial distributions and temporal dynamics of electrical activity of the brain.

\section{Methods and Experiment}

\subsection{Materials}

The study involved two groups of subjects: group A, 4 children aged 9-10 years with vision impairment up to $25 \%$; group B, 4 children aged 9-10 years with normal vision (90-100\%). The criteria for exclusion from the study were severe neurological disorders. Moreover, every child underwent preliminary psychological tests in order to exclude cognitive deficit problems and psycho-emotional instability. The experiment was built around audio stimulation. Thus, the group of children was not supposed to have any problems related to cognitive tasks and attention tasks in the course of the experiment. Two subjects that demonstrated certain individual peculiarities in the evoked potentials, component $\mathrm{P}$ (300) specifically, were selected from the preliminary group based on the experiment results. To follow is demonstration of the detailed study of their abilities, as well as the statistical comparison.

EEG data in the course of the experiment was recorded with the electroencephalograph (MTD "Medikom", Russian Federation), 21 active EEG channels with a temporal resolution of $250 \mathrm{~Hz}$ according to the standard scheme "10 - 20" [17]. All the experiments were carried out in the darkened room with shades on the windows to prevent direct light access. The subject was seated in a comfortable armchair individually adjusted for him/her. The experiment duration for every child was about 25 minutes. EEG recording included the state of calm wakefulness with eyes closed at the beginning and at the end of the experiment, as well as taking a cognitive test, which involved counting repetitive groups of short sound stimuli with rest pauses. The duration of the stages of recording quiet wakefulness was 3 minutes each. The duration of the cognitive test involved 3 stages of active counting 3 minutes long each and 4 stages of rest 2 minutes each. Short sound stimuli came in groups from 1 to 4 in a pseudo-random order, which was the same for all subjects. The goal was to indicate an even or odd number of stimuli in the group. For each group of stimuli the subject pressed the corresponding left or right button on the control panel, that a child was holding in his/her hands. The groups of sound stimuli were separated by pauses, with randomly set duration within the range of 5 to 8 seconds. In the automated experiment environment 
protocols with the moments of presentation of each stimulus were compiled automatically and subsequently used to build the evoked potentials.

Experimental studies were carried out in accordance with the ethical standards [18] and approved by the local committee of research ethics under the auspices of Saratov State University.

\subsection{Methods}

Evoked potentials have long had a good track record in the EEG data analysis tasks when assessing a subject's response to external visual or auditory stimuli [11]. This method of analysis is suitable for cases when multiple identical or similar stimuli are presented $[4,10]$. Upon the completion of the experiment, averaging over the time series can be performed after stimulus presentation. In this case, it is important to determine the end of the stimulus accurately. If for a given channel the stimulus evokes a similar response in all stimulus presentations, then after averaging the signal amplitude increases, while the random component of the signal tends to zero. Thus, the evoked potentials are convenient for singling out the EEG features under certain types of stimulation, for example, as in the experiment that was conducted.

It is important to point out that different stimuli elicit different evoked potentials. The differences in the evoked potentials involve different location of the potential components (its extrema) over the period of time. Thus, cognitive evoked potentials are released by the $\mathrm{P}$ (300) component - that is, clearly distinguished by the maximum N2 and minimum P3, which are typically located near the time mark of $300 \mathrm{~ms}$. This position of the $\mathrm{P}$ (300) component is common for all people (within the framework of statistics). However, some individual peculiarities are also possible (especially against the background of impaired cognitive activity); they lead to a shift in the time intervals at which these components are observed.

Within the experimental paradigm, we calculated the evoked potential of the subject from the point of completion of the last sound stimulus in the group. Figure 1 shows the evoked potentials calculated for a pair of subjects from groups A and B according to the EEG data from the Fz channel. Cognitive evoked potentials are often best identified along the central axis of the brain, rather than its hemispheres [15]. We can see that for the given subjects, the $\mathrm{N} 1$ and P2 components lie within close time intervals. In this case, the rest of the evoked potentials' components are shifted along the time scale, including the P (300) component. Thus, we can clearly see strong changes in cognitive evoked potentials for these subjects.

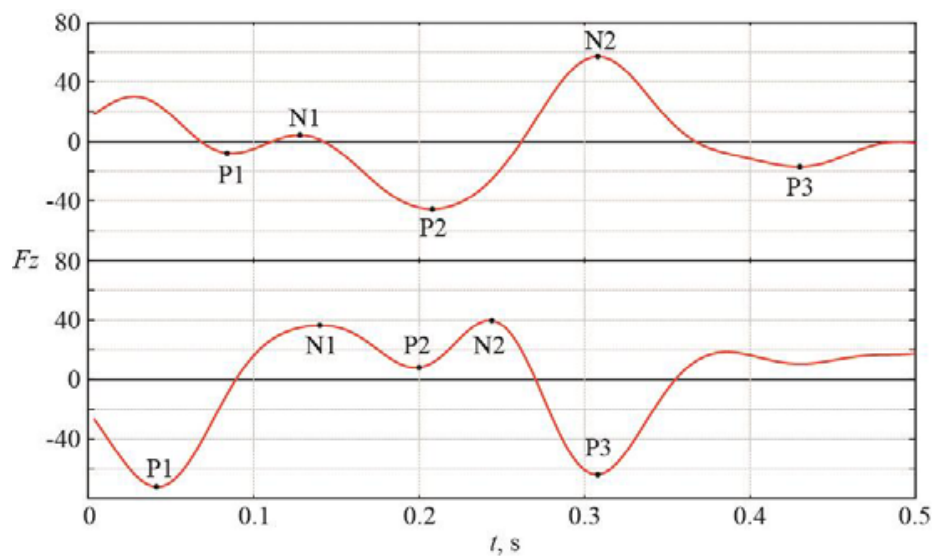

Fig. 1. Examples of built potentials for two subjects in the Fz channel with data for individual components presented as points. Above - the results for group A subject, below - the results for group B subject. 


\section{Results}

In this study we compared individual features of cognitive evoked potentials, which subjects with normal and significantly impaired vision experience. We carried out analysis of the spatio-temporal dynamics of evoked potentials across various registration channels and statistical analysis of the similarity of time series according to the Wilcoxon test.

\subsection{Analysis of temporal dynamics across the channels}

It is convenient to draw up a chart of the subjects' head map with evoked potential's amplitude marks for the channel with the fixed step size in time using the specialized FieldTrip package (MathLab software environment). We used a step size of $50 \mathrm{msec}$ to create the head map for the time interval $[50,450]$ msec. We chose the time interval and step size based on the convenience of observing components of the cognitive evoked potential. Fig. 2 and Fig. 3 show the results for the subjects from groups A and B, respectively. Calculation results correspond to the upper evoked potential shown in Fig.2.
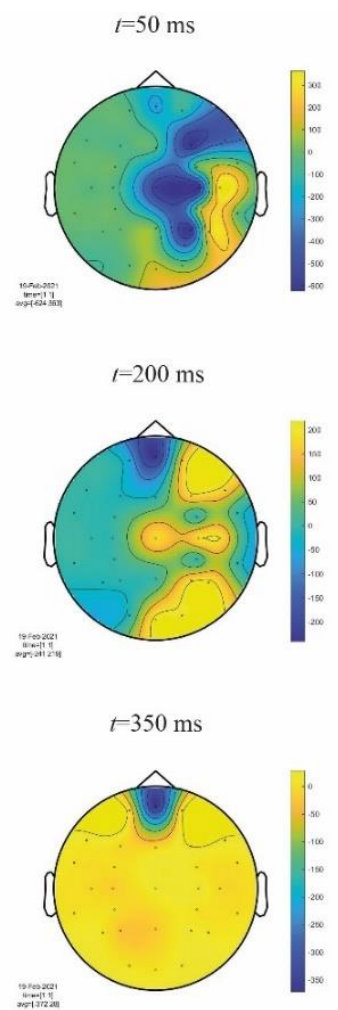
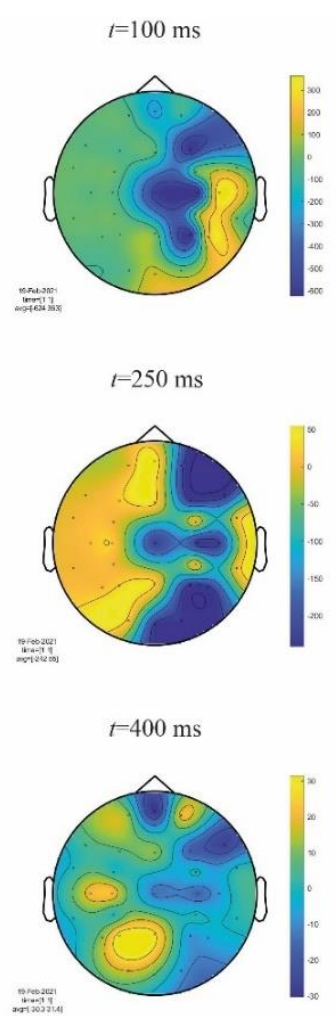
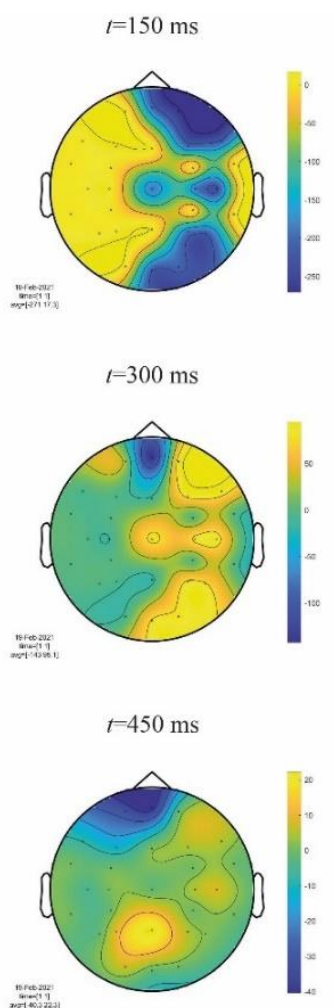

Fig. 2. Time slices of the evoked potentials plotted on the spatial chart of the subject's head from group A.

Fig. 2 shows the map of the subject's head from group A, whose evoked potential is shown in the channel Fz at the top of Fig. 1. First of all, it should be noted that for this subject the main changes in the values of the evoked potential are observed in the right side of the brain. Maximum deviations from zero are registered in this area except for the timepoints $\mathrm{t}=350 \mathrm{msec}$ and $\mathrm{t}=450 \mathrm{msec}$. Furthermore, the value of the evoked potential is close to zero 
in the left side of the brain, except for the timepoint $\mathrm{t}=400 \mathrm{msec}$. We observed the maximum values in the left side and the minimum values in the right side at that moment.

We can track the precise timepoints at which we observe the maximum or the minimum value in the dynamics of the components of the cognitive evoked potential in the right side of the brain. We observed the first significant minimum at the timepoint $t=150 \mathrm{msec}$ (it corresponds to component P1), further at the timepoint $\mathrm{t}=250 \mathrm{msec}$ (component $\mathrm{P} 2$ ), and insignificant minimum at the timepoint $\mathrm{t}=400 \mathrm{msec}$ (component $\mathrm{P} 3$ ). The maximum values, that correspond to components $\mathrm{N} 1$ and N2, are marked at the timepoints $\mathrm{t}=200 \mathrm{msec}$ and $\mathrm{t}=300 \mathrm{msec}$, respectively. The timepoints correspond to overall component latency for cognitive evoked potentials $[15,16]$. Therefore, having completed the analysis of the temporal dynamics of a given subject, we came to the conclusion that components of the evoked potentials for him/her corresponded well to the mean values but only for the right side of the brain. Evoked potentials were very faintly expressed in the left side of the brain.
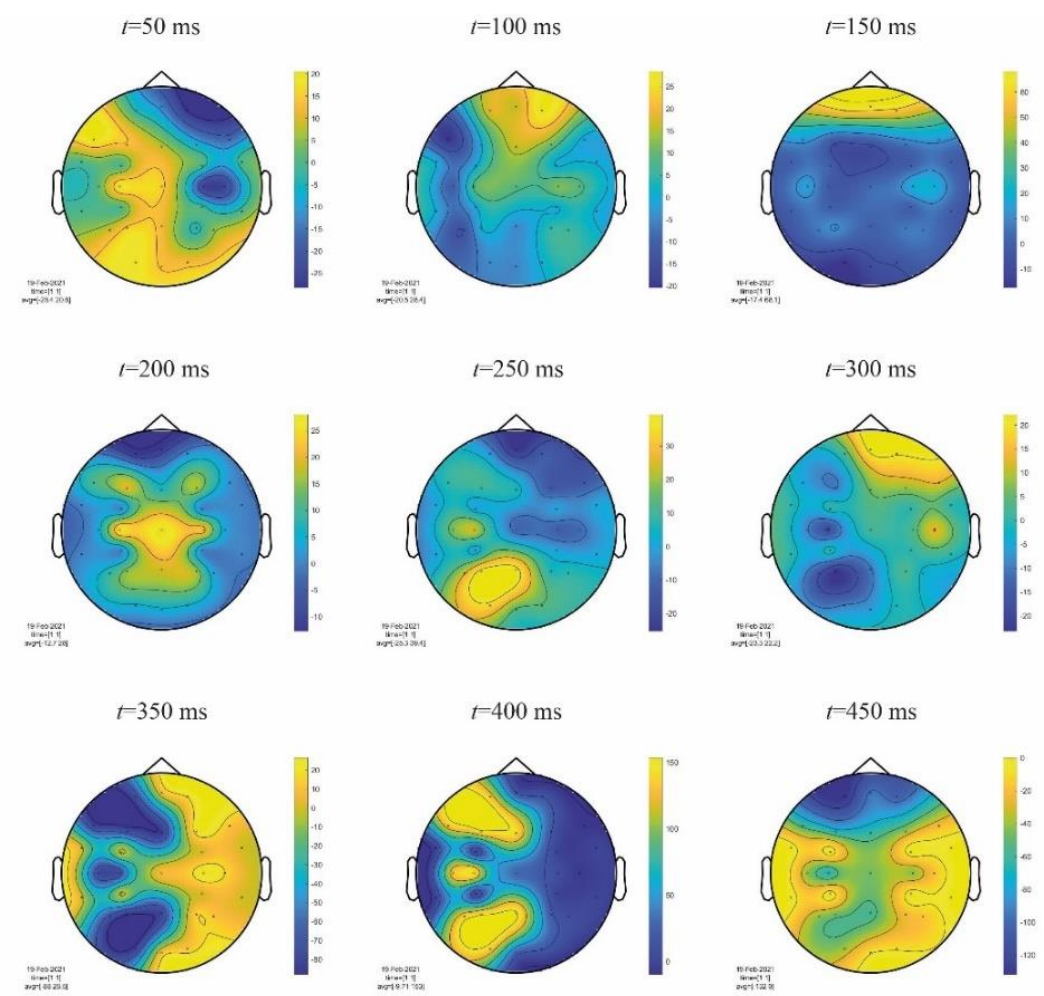

Fig. 3. Time slices of the evoked potentials plotted on the spatial chart of the subject from group B, which correspond to the lower evoked potential shown in Fig. 1.

Moving on to group B we should point out that it is logical to observe the brain of the subject from group B divided into the frontal lobe and the rest of the lobes. We observe minimum values in the frontal lobe at the timepoints $\mathrm{t}=50 \mathrm{msec}, \mathrm{t}=200-250 \mathrm{msec}$ and $\mathrm{t}=400$ $450 \mathrm{msec}$. Another timepoints are characterized by the maximum value of the evoked potential. The value of the evoked potentials for other parts of the brain acts in the opposite way. Therefore, we can state that evoked potentials in the frontal lobe of the subject go ahead or lag behind at 50-100 msec compared to the other sides. Consequently, it leads to the fact that it is difficult to determine overall latency for a given subject and correlate it with the average indicators for the adult subjects, respectively. It is curious that we can observe 
separation of the values of the evoked potentials in the left and right parts of the brain for a given subject at the timepoints $\mathrm{t}=350 \mathrm{msec}$ and $\mathrm{t}=400 \mathrm{msec}$.

\subsection{Statistical analysis}

Having described some individual peculiarities of the subjects, based on the analysis of the temporal dynamics of the evoked potentials, we would like to turn to statistical analysis. For this purpose, we carried out statistical assessment of the evoked potentials in the two groups of subjects across different channels using the Wilcoxon test to achieve the goal. Table 1 shows the analysis results.

Table 1. Validity of differences statement featuring the evoked potentials in subjects from groups A and $B$ tested across the channels according to the Wilcoxon test $(p<0.05)$.

\begin{tabular}{|c|c|c|c|c|c|c|c|}
\hline Chanel & 'O2' & 'O1' & 'P4' & 'P3' & 'C4' & 'C3' & 'F4' \\
\hline Value & 0.05447 & $\mathbf{0 . 0 3 5 5 6}$ & $\mathbf{0 . 0 0 6 3 7}$ & $\mathbf{0 . 0 1 3 9 0}$ & 0.06671 & $\mathbf{0 . 0 0 6 3 7}$ & 0.11752 \\
\hline Chanel & 'F3' & 'Fp2' & 'Fp1' & 'T6' & 'T5' & 'T4' & 'T3' \\
\hline Value & $\mathbf{0 . 0 2 2 5 6}$ & 0.30367 & 0.68732 & 0.62276 & $\mathbf{0 . 0 1 3 9 0}$ & $\mathbf{0 . 0 0 1 2 6}$ & 0.00149 \\
\hline Chanel & 'F8' & 'F7' & 'Oz' & 'Pz' & 'Cz' & 'Fz' & 'Fpz' \\
\hline Value & 0.30367 & $\mathbf{0 . 0 0 1 4 9}$ & 0.26355 & 0.09798 & $\mathbf{0 . 0 0 0 2 9}$ & 0.11752 & 0.75424 \\
\hline
\end{tabular}

The channels that meet the Wilcoxon test criteria are in bold and coloured grey in the table. It should be pointed out that most of the channels belong to the left side of the brain (O1, P3, C3, F3, T5, F7). Only two channels, P4 and T4, are located in the right side and channel $\mathrm{Cz}$ is located in the central part of the longitudinal fissure projection.

\section{Discussion and conclusion}

In this work we have used psychophysiological assessments of the human brain electrical activity according to the classical neurological method for calculating the evoked potential. Today, the evoked potential analysis is used to assess cognitive functions and the nervous system of adult patients with neurological disorders. Analysis of the evoked potentials, which can be observed in children of different ages, is described in scientific works, however, it has not yet become part of the clinical assessment standards. We carried out analyses described in the present study to solve this problem.

We used auditory stimuli under EEG monitoring control to perform "loading" of the cognitive function of a child's attention. Assessment of cognitive evoked potentials for a countable auditory stimulus shows that the characteristics of brain activity change in children with significant vision impairment. Interestingly enough, we observed significant latency in the evoked potential distributions when significant differences between children with normal vision and significantly reduced vision are concentrated in the left side of the brain. We already know a lot about interhemispheric asymmetry in the brain activity, which is recorded during EEG monitoring, and other approaches to neuroimaging. For example, Luschekina and et al., Dickinson and et al. establish similar patterns for children with autism spectrum disorder in spectral EEG characteristics [19, 20]. Furthermore, it has been demonstrated how the analysis of interhemispheric asymmetry of brain bound states at rest is correlated with the development of cognitive function of spatial perception [21].

Thus, in this work we demonstrated interhemispheric change in a simple clinically tested characteristic of the evoked potentials for children with visual impairment for the first time. Analysis of such features, primarily in comparison with average values for conditionally healthy subjects, becomes an important goal when using BCI-systems when solving practical 
problems of developing rehabilitation systems for patients with various disabilities. The sequence of evoked potential analysis demonstrated in the article suits for adjusting the settings of the "brain-computer" systems for a particular subject and allows to select channels used in further BCI training efficiently.

\section{Acknowledgement}

This work was supported by Ministry of Science and Higher Education of the Russian Federation in the framework of the state assignment (project No. FSRR-2020-0003).

\section{References}

1. S. Broyd, S. Debener, Neurosci Biobehav Rev. 33, 279296 (2009)

2. S. Baillet, R.M. Leahy, IEEE Signal Processing Magazine 18, 10535888 (2001)

3. M.F. Bear, B.W. Connors, M.A. Paradiso, Neuroscience Exploring the Brain (Woters Kluwer, Netherlands, 2015)

4. N.R. Cooper, A.P. Burgess et al., NeuroReport 17, 205-208 (2006)

5. V.A. Maksimenko, A.E. Runnova et al., PloS one 13(9), e0197642 (2018)

6. A.N. Pisarchik, R. Jaimes-Reátegui, C.D.A. Magallón-García et al., Biological Cybernetics 108(4), 397404 (2014)

7. A.E. Hramov, A.A. Koronovskii, V.A. Makarov et al., Wavelets in Neuroscience. Springer Series in Synergetics (Springer, Heidelberg, New York, 2015)

8. S.D. Puthankattil, P.K. Joseph, R.U. Acharya, Journal of Medical Systems 34, 195212 (2010)

9. R. Acharya, N. Kannathal et al., Computer Methods and Programs in Biomedicine 80, 3745 (2005)

10. S.A. Hillyard, H. Hinrichs, C. Tempelmann et al., Hum. Brain Mapp. 5, 287-292 (1997)

11. S. Makeig, M. Westerfield, T.P. Jung et al., Science 295, 690-694 (2002)

12. J. Mast, J.D. Victor, Clin. Neurophysiol. 78, 389-401 (1993)

13. B. Schack, W. Klimesch, Neurosci. Lett. 331, 107-110 (2002)

14. L. Parra, C. Spence, A. Gerson, P. Sajda, IEEE Trans. on Neural Systems and Rehabilitation Engineering 11(2), 173-7 (2003)

15. M.-W. Huang, F.H.-C. Chou, P.-Y. Lo, K.-S. Cheng, BMC Psychiatry 11, 74 (2011)

16. E.V. Levichkina, A.Ya. Kaplan, Human Physiology 35(2), 74 (2009)

17. V. Jurcak, D. Tsuzuki, I. Dan, Neuroimage 34(4), 27-32 (2007)

18. World medical association, The Journal of the American Medical Association 284(23), 30433045 (2000)

19. E.A. Luschekina, O.Y. Khaerdinova, V.S. Luschekin, V.B. Strelets, Human Physiology 43(3), 265-273 (2017)

20. A. Dickinson, C. Di Stefano, Y.Y. Lin et al, Behavioural brain research 348, 227-234 (2018)

21. Z. Gracia-Tabuenca, M.B. Moreno, F.A. Barrios, S. Alcauter, NeuroImage 174, 441-448 (2018) 